\title{
Energy Conditions and Junction Conditions
}

\author{
Donald Marolf \\ Physics Department, UCSB, \\ Santa Barbara, CA 93106, USA \\ and \\ Perimeter Institute, 31 Caroline St. N, \\ Waterloo, Ontario N2L 2Y5, Canada \\ marolf@physics.ucsb.edu \\ Sho Yaida \\ Mathematics Department, UCSB, \\ Santa Barbara, CA 93106, USA \\ yaida@umail.ucsb.edu \\ (Dated: August, 2005)
}

\begin{abstract}
We consider the familiar junction conditions described by Israel for thin timelike walls in EinsteinHilbert gravity. One such condition requires the induced metric to be continuous across the wall. Now, there are many spacetimes with sources confined to a thin wall for which this condition is violated and the Israel formalism does not apply. However, we explore the conjecture that the induced metric is in fact continuous for any thin wall which models spacetimes containing only positive energy matter. Thus, the usual junction conditions would hold for all positive energy spacetimes. This conjecture is proven in various special cases, including the case of static spacetimes with spherical or planar symmetry as well as settings without symmetry which may be sufficiently well approximated by smooth spacetimes with well-behaved null geodesic congruences.
\end{abstract}

\section{INTRODUCTION}

The study of boundary layers (i.e., singular sheets of sources with zero thickness) is of longstanding interest in both electromagnetism and general relativity. In addition to approximating smooth solutions such as domain walls, the class of "thin-wall" solutions provides a useful laboratory in which to explore new phenomena. For example, thin-wall spacetimes have been of much use in investigating so-called "braneworld" scenarios, first suggested in the modern context in [1, 2, 3] , in which the visible $3+1$ universe is confined to a submanifold of some higher dimensional spacetime. In particular, the phenomenon by which gravity can become localized along such a domain wall was discovered by Randall and Sundrum [4, 5] by considering the limit of an infinitely thin wall. Thin-wall spacetimes are also historically of interest in exploring gravitational collapse (see e.g. [6]), typically in the context of spherical shells.

Although the stress tensor must diverge at an infinitely thin wall, the associated singularities in Einstein-Hilbert gravity are often mild. For familiar cases, these singularities serve merely to simplify the equations of motion by turning the differential equations for the fields into finite difference equations, known as "junction conditions," governing the discontinuities of various fields across the thin wall. The standard formalism for treating thin walls with spacelike normals was developed by Israel 7], building on the work of others (e.g., 8, 9, 10, 11]).

Briefly, the Israel formalism 7] considers spacetimes with a distinguished singular hypersurface $\Sigma^{0}$ of co-dimension one. One imagines foliating the spacetime near $\Sigma^{0}$ in an arbitrary way such that $\Sigma^{0}$ is one of the hypersurfaces in the foliation. One then requires that both the induced metric $h$ and the extrinsic curvature $K$ of hypersurfaces in this foliation have well-defined limits $h_{ \pm}, K_{ \pm}$as the singular hypersurface $\Sigma^{0}$ is approached from each side. Furthermore, one requires that the induced metric is continuous across $\Sigma^{0}: h_{+}=h_{-}$. In such a setting, Israel showed that the remaining junction condition for a wall with spacelike normal is

$$
[K]_{i j}=8 \pi G_{N}\left(S_{i j}-\frac{h_{i j}}{d-2} h^{k l} S_{k l}\right)
$$

Here $[K]_{i j}=K_{+i j}-K_{-i j}$ is the discontinuity in the extrinsic curvature across the surface and $S_{i j}$ is the so-called surface stress tensor, which is essentially the pull-back of the stress-energy tensor integrated over a small region around the hypersurface $\Sigma^{0}$. We work in $d$ spacetime dimensions (so that the wall has $(d-1)$ spacetime dimensions) and $G_{N}$ is Newton's gravitational constant. In this formalism, components of the stress tensor not captured by $S_{i j}$ remain bounded at the wall, so that the tensor $S_{i j}$ captures the "delta-function part" of the stress-energy tensor.

Since condition (1.1) follows from the Einstein equation integrated over a small region, it is firmly grounded in physics. Consider, however, the more fundamental requirement of continuity of the induced metric: $h_{+}=h_{-}$. In textbooks, this requirement is often motivated by aesthetic concerns, such as the desire for $\Sigma^{0}$ to have a well-defined 
induced geometry, or the related desire to have a simple description of the dynamics of particles bound to $\Sigma^{0}$. Another motivation is that the ansatz $h_{+}=h_{-}$does in fact allow one to solve the Einstein equation (via (1.1)) whenever the singular part of the stress tensor is captured by $S_{i j}$. What remains unclear, however, is whether this requirement is in fact satisfied by all cases of physical interest.

Israel's own motivations [12] for imposing the continuity of $h$ are somewhat more enlightening, and were based on an analogy with electromagnetism (in particular, with electrostatics). In the electrostatic context, one finds that a discontinuity in the component $E_{\perp}$ of the electric field orthogonal to the surface is associated with a surface charge density $\sigma$ in direct correspondence with (1.1). In contrast, a discontinuity in the parallel components $E_{\|}$of the electric field is associated with a distribution of dipole sources on the surface. As one expects no gravitational point dipoles, one might also expect the gravitational field (and in particular the induced metric) to be continuous across the surface. Of course, the reason that one expects no gravitational dipoles is that point dipoles require both positive and negative charges.

Our purpose in the present work is to explore the conjecture (implicit in the above reasoning) that taking thinwall limits of spacetimes satisfying an appropriate positive energy condition will necessarily lead to continuity of the induced metric $h$, and thus to the familiar condition (1.1). Such a conjecture is non-trivial due to the intrinsic non-linearities of general relativity. Indeed, since the curvature diverges at thin walls, one expects non-linearities to have significant effects.

Let us take a few moments to make the notion of a "thin-wall limit" more precise. To do so, consider a oneparameter family of smooth Lorentz-signature metrics $g_{\lambda}$ on a fixed manifold $M$ for $\lambda \in(0,1]$. Thus, each $\left(M, g_{\lambda}\right)$ is a spacetime. However, we think of each $g_{\lambda}$ as merely some tensor field on the manifold $M$, where in particular the differential structure of $M$ is fixed and independent of $\lambda$. We require the spacetime dimension of $M$ to be $d \geq 3$ so that the Einstein equation contains non-trivial dynamics.

In the limit $\lambda \rightarrow 0$, we wish to allow a thin wall to develop on some hypersurface; that is, on a smooth embedded submanifold of co-dimension one, say $\Sigma^{0}$. Note that, while we require $\Sigma^{0}$ to be smooth with respect to the differentiable

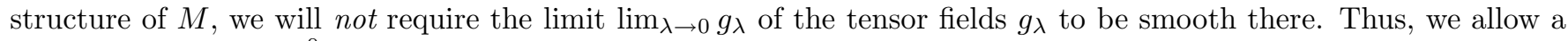
thin wall to form at $\Sigma^{0}$ as one decreases the width of any "thick wall" which may be present for $\lambda \neq 0$.

However, we will require the limiting tensor field to be smooth away from $\Sigma^{0}$ so that we encounter only thin-wall singularities. In particular, we suppose that our family $g_{\lambda}$ satisfies the following conditions:

i) The limit of $g_{\lambda}$ as $\lambda \rightarrow 0$ defines a smooth Lorentz-signature metric $g_{0}$ everywhere away from $\Sigma^{0}$. Similarly, we require that the first and second derivatives of $g_{\lambda}$ (with respect to any fixed smooth coordinate) converge to the first and second derivatives of $g_{0}$ everywhere on $M$ away from $\Sigma^{0}$. For simplicity, we have in mind taking the pointwise limit of the components of each tensor field $g_{\lambda}$ and of its derivatives. Thus, this assumption restricts not only the geometry associated with $g_{\lambda}$, but also the inherent freedom to perform diffeomorphisms as a function of $\lambda$. Note that we do not require $\left(M, g_{\lambda}\right)$ to be in any sense complete, so that $M$ may in fact represent a small region of a larger manifold and other singularities could arise elsewhere.

ii) Each $g_{\lambda}$ with $\lambda>0$ satisfies the null convergence condition: $R_{a b} k^{a} k^{b} \geq 0$ for all null $k^{a}$. Here our conventions are those of [13].

Note that, without condition (iii), it is straightforward to construct a family $g_{\lambda}$ satisfying (ii) such that the induced metric in the limit $\lambda \rightarrow 0$ is discontinuous across the wall. For example, one may consider the following metrics on $\mathbb{R}^{d}$ :

$$
d s_{\lambda}^{2}:=\left(g_{\lambda}\right)_{a b} d x^{a} d x^{b}=-f_{\lambda}(z) d t^{2}+d z^{2}+\sum_{i=1}^{d-2}\left(d x^{i}\right)^{2},
$$

where $f_{\lambda}$ is some smooth (positive) function with $f_{\lambda}(z)=1$ for $z \leq-\lambda$ and $f_{\lambda}(z)=2$ for $z \geq+\lambda$. This one-parameter

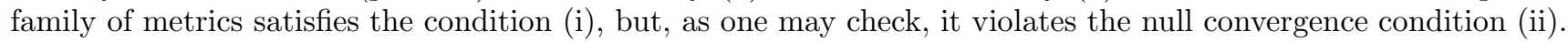

Our goal is to investigate what further technical assumptions must be added to (1i) and (iii) in order to guarantee that $g_{0}$ defines an induced metric on $\Sigma^{0}$ which is continuous across the wall. While a completely general theorem is beyond the scope of this work, we present some partial results below. In section II we prove a number of results to the effect that, in the context of various ansätze for the metrics $g_{\lambda}$, the conditions (ii) and (iii) do indeed imply that $g_{0}$ defines an induced metric which is continuous across $\Sigma^{0}$. In particular, section $\amalg A$ considers a case where one assumes translation invariance along the surface $\Sigma^{0}$ as well as rotation symmetry among the spatial directions of $\Sigma^{0}$. This case may also be viewed as the warped product of a real line with another real line (the time direction) and with a Euclidean plane, where warping of the second and third factors is allowed only with respect to the first real line. This simple setting also serves to introduce a few technical tools (Lemmas) which will continue to be of use later in 
section IIB where we allow the plane in the above warped product to be replaced with an arbitrary geometry; i.e., we generalize to metrics of the form

$$
d s^{2}=-e^{2 \alpha(z)} d t^{2}+d z^{2}+e^{2 \beta(z)} \sum_{i, j=1}^{d-2} \tilde{g}_{i j}(x) d x^{i} d x^{j},
$$

where $x=\left(x^{1}, \ldots, x^{d-2}\right)$ is an appropriate set of spatial coordinates on the hypersurfaces of the foliation. In particular, any given static, spherically symmetric metric may be written in the form (1.3). For this case, we also introduce a further assumption guaranteeing that the wall is in fact "thin" in a sense measured by certain null geodesics. A final static special case with more degrees of freedom (but of less physical interest) is studied in section

In contrast, section $\amalg$ pursues a different approach. There we prove a theorem which requires neither symmetry nor a particular ansatz for the metric. However, the price to be paid is the introduction of a number of detailed assumptions which relate the behavior of null geodesic congruences in the spacetime $\left(M, g_{0}\right)$ to that of null geodesic congruences in $\left(M, g_{\lambda}\right)$. We also assume that the induced metric on each side of the thin wall is well-defined, whereas we were able to derive this result within the context of section [I] Finally, section $\amalg$ requires the induced metric on each side to be timelike. With these additional assumptions, the Raychaudhuri equation leads directly to continuity of the induced metric. Through its use of the Raychaudhuri equation, this theorem gives some physical insight into how the null convergence condition helps to ensure this continuity. We then close with a brief discussion in section IV In particular, we emphasize that if one does not impose a positive energy condition (e.g., as in the Randall-Sundrum scenario [4, [5]), then there is in general no reason to expect the induced metric to be continuous across the junction.

\section{PROOFS OF THE CONJECTURE IN SPECIAL CASES}

We now turn to proofs of the above conjecture in various special cases. We shall begin by considering (section 【A a special case of the setting laid out in section $\square$ above which has a particularly high degree of symmetry. This will simplify the algebra involved and allow us to introduce two Lemmas in a context where their use is transparent. We then proceed in section IIB to a more general static case involving a warped product, as would be appropriate for spherical symmetry. In section $\llbracket \mathrm{C}$ we briefly examine a further special case with more degrees of freedom, but which is of less physical interest as it requires a timelike Killing field of constant norm.

\section{A. Translation and Spatial rotation invariance: a simple first case}

We begin with the case where the metrics $g_{\lambda}$ are static and share a common set of Euclidean (i.e., rotational and translational) symmetries. We further assume that Gaussian normal coordinates in $\left(M, g_{\lambda}\right)$ based on the hypersurface $\Sigma^{0}$ are independent of $\lambda$. Thus, our metrics take the form:

$$
d s_{\lambda}^{2}=-e^{2 \alpha_{\lambda}(z)} d t^{2}+d z^{2}+e^{2 \beta_{\lambda}(z)} \sum_{i=1}^{d-2}\left(d x^{i}\right)^{2},
$$

where $x=\left(x^{1}, \ldots, x^{d-2}\right)$ provides an appropriate coordinate system on the $(d-2)$-planes which form the orbits of the Euclidean symmetry. This case includes, for example, the setting studied by Randall and Sundrum [4, [5]. For definiteness, we take the range of $z$ to include the $\lambda$-independent closed interval $\left[z_{-}, z_{+}\right]$with $z_{+}>0>z_{-}$, and we will always work on this closed interval below.

Here, condition (1i) of section प merely imposes that $\alpha_{\lambda}, \beta_{\lambda}$, and their first and second derivatives converge for $z \neq 0$ to some $\alpha_{0}, \beta_{0}$ (and their derivatives) in the limit $\lambda \rightarrow 0$. On the other hand, requirement (iii) imposes the null convergence condition for each $\lambda$. Considering the null vectors

$$
\begin{aligned}
k_{\perp} & =e^{-\alpha_{\lambda}} \partial_{t}+\partial_{z}, \\
k_{\|} & =e^{-\alpha_{\lambda}} \partial_{t}+e^{-\beta_{\lambda}} \partial_{x_{1}},
\end{aligned}
$$

one finds:

$$
\begin{aligned}
& 0 \leq R_{a b} k_{\perp}^{a} k_{\perp}^{b}=(d-2)\left[-\beta_{\lambda}^{\prime \prime}-\beta_{\lambda}^{\prime 2}+\alpha_{\lambda}^{\prime} \beta_{\lambda}^{\prime}\right], \quad \text { and } \\
& 0 \leq R_{a b} k_{\|}^{a} k_{\|}^{b}=\alpha_{\lambda}^{\prime \prime}+\alpha_{\lambda}^{\prime 2}+(d-3) \alpha_{\lambda}^{\prime} \beta_{\lambda}^{\prime}-\beta_{\lambda}^{\prime \prime}-(d-2) \beta_{\lambda}^{\prime 2} .
\end{aligned}
$$


We now wish to prove continuity of $\alpha_{0}, \beta_{0}$ at $z=0$. The essential strategy will be to use (2.3), (2.4) and the convergence of $\alpha_{\lambda}, \beta_{\lambda}$ to $\alpha_{0}, \beta_{0}$ (for $z \neq 0$ ) to show that the derivatives $\alpha_{\lambda}^{\prime}, \beta_{\lambda}^{\prime}$ at $z=0$ satisfy a bound that is independent of $\lambda$ for small $\lambda$. Thus, even the limiting functions $\alpha_{0}, \beta_{0}$ must be continuous at $z=0$.

However, before commencing our main argument, we first note that condition (2.3) restricts the possible local extrema of $\beta_{\lambda}$. Indeed, since the first derivative vanishes at such an extremum, we find $\beta_{\lambda}^{\prime \prime} \leq 0$. If $\beta_{\lambda}^{\prime \prime}$ is non-vanishing there, the extremum must be a local maximum.

In fact, the following argument shows that there are no local minima even in the case where $\beta_{\lambda}^{\prime \prime}$ vanishes at an extremum. Suppose $\beta_{\lambda}$ satisfies (2.3) and has a local minimum at $z_{\text {min }}$. Then $\beta_{\lambda}^{\prime}$ is negative somewhere before $z_{m i n}$, and we may in fact choose an open interval on which $\beta_{\lambda}^{\prime}$ is negative, but such that $\beta_{\lambda}^{\prime}$ approaches zero at the right end of this interval ${ }^{1}$. On that open interval we may write (2.3) as

$$
0 \leq \frac{1}{\left(-\beta_{\lambda}^{\prime}\right)}\left[-\beta_{\lambda}^{\prime \prime}-\left(\beta_{\lambda}^{\prime}\right)^{2}+\alpha_{\lambda}^{\prime} \beta_{\lambda}^{\prime}\right]=\frac{\beta_{\lambda}^{\prime \prime}}{\beta_{\lambda}^{\prime}}-\left(\alpha_{\lambda}^{\prime}-\beta_{\lambda}^{\prime}\right)=\frac{d}{d z}\left(\log \left(-\beta_{\lambda}^{\prime}\right)\right)-\left(\alpha_{\lambda}^{\prime}-\beta_{\lambda}^{\prime}\right) .
$$

But $\beta_{\lambda}^{\prime}$ vanishes at the endpoint, so $\log \left(-\beta_{\lambda}^{\prime}\right)$ diverges toward negative infinity. On the other hand, since $\alpha_{\lambda}$ and $\beta_{\lambda}$ are smooth functions, the remaining term $\alpha_{\lambda}^{\prime}-\beta_{\lambda}^{\prime}$ remains bounded. Thus, we have reached a contradiction and $\beta_{\lambda}$ can have no local minima. A similar argument using equation (2.4) in the form

$$
-\frac{d^{2}}{d z^{2}}\left(\beta_{\lambda}-\alpha_{\lambda}\right)+\left(\beta_{\lambda}^{\prime}-\alpha_{\lambda}^{\prime}\right)\left[-\alpha_{\lambda}^{\prime}-(d-2) \beta_{\lambda}^{\prime}\right] \geq 0
$$

shows that the function $\beta_{\lambda}-\alpha_{\lambda}$ can have no local minima.

Let us summarize these results and some of the implications in the following Lemma:

Lemma 1: Suppose that, for some $\lambda$, equations (2.3) and (2.4) hold on an interval $\left[z_{-}, z_{+}\right]$and that $\alpha_{\lambda}, \beta_{\lambda}$ are smooth on this interval. Then we have the lower bounds $\beta_{\lambda} \geq \min \left\{\beta_{\lambda}\left(z_{-}\right), \beta_{\lambda}\left(z_{+}\right)\right\}$and $\beta_{\lambda}-\alpha_{\lambda} \geq \min \left\{\left(\beta_{\lambda}-\alpha_{\lambda}\right)\left(z_{-}\right),\left(\beta_{\lambda}-\alpha_{\lambda}\right)\left(z_{+}\right)\right\}$everywhere on this interval.

Now, as a result of the assumption that the $\beta_{\lambda}$ converge to $\beta_{0}$ for $z \neq 0$, the values $\beta_{\lambda}\left(z_{ \pm}\right)$are close to $\beta_{0}\left(z_{ \pm}\right)$for small $\lambda$ (and similarly for $\beta_{\lambda}-\alpha_{\lambda}$ ). Thus we also have

Corollary 1: The functions $\beta_{\lambda}$ and $\beta_{\lambda}-\alpha_{\lambda}$ are bounded below on the interval $\left[z_{-}, z_{+}\right]$, uniformly in $\lambda$ for small $\lambda$. As a direct consequence, for small $\lambda$ the functions $e^{-\beta_{\lambda}}$ and $e^{\alpha_{\lambda}-\beta_{\lambda}}$ are bounded (uniformly in $\lambda)$ both above and below.

To simplify the remaining analysis, it is useful to introduce the following two quantities:

$$
A_{\lambda}:=-\beta_{\lambda}^{\prime} e^{\beta_{\lambda}-\alpha_{\lambda}}, \quad B_{\lambda}:=\left[\frac{d}{d z}\left(e^{\alpha_{\lambda}-\beta_{\lambda}}\right)\right] e^{(d-1) \beta_{\lambda}} .
$$

With these definitions, (2.3) and (2.4) are equivalent to:

$$
0 \leq \frac{d}{d z}\left[A_{\lambda}\right] \text { and } \quad 0 \leq \frac{d}{d z}\left[B_{\lambda}\right]
$$

so that both $A_{\lambda}$ and $B_{\lambda}$ are non-decreasing. In particular, $A_{\lambda}\left(z_{-}\right) \leq A_{\lambda}(z) \leq A_{\lambda}\left(z_{+}\right)$for $z \in\left[z_{-}, z_{+}\right]$, and similarly for $B_{\lambda}$. Since, for small $\lambda, A_{\lambda}\left(z_{ \pm}\right)$is close to $A_{0}\left(z_{ \pm}\right)$and $B_{\lambda}\left(z_{ \pm}\right)$is close to $B_{0}\left(z_{ \pm}\right)$, we arrive at the following Lemma:

Lemma 2: The functions $A_{\lambda}$ and $B_{\lambda}$ are bounded both above and below on the interval $\left[z_{-}, z_{+}\right]$. Furthermore, for sufficiently small $\lambda$, one may choose bounds that are independent of $\lambda$.

The proof of the conjecture now follows by combining Corollary 1 and Lemma 2 . We see that both $\beta_{\lambda}^{\prime}=-A_{\lambda} e^{\alpha_{\lambda}}-\beta_{\lambda}$ and $\frac{d}{d z}\left(e^{\alpha_{\lambda}-\beta_{\lambda}}\right)=B_{\lambda} e^{-(d-1) \beta_{\lambda}}$ are bounded uniformly in $\lambda$ for small $\lambda$, so that $\beta_{0}, e^{\alpha_{0}-\beta_{0}}$, and therefore $e^{\alpha_{0}}$ must be continuous. Let us summarize the result in the following Theorem:

Theorem 1: For a one-parameter family of smooth metrics described by the ansatz (2.1) and satisfying conditions (1i) and (iil) from section (1) the limiting metric $g_{0}$ defines continuous induced metrics on the hypersurfaces $\Sigma^{z}$.

Thus, we have proven our conjecture for the special case described by the ansatz (2.1).

\footnotetext{
1 This endpoint need not be $z_{m i n}$.
} 


\section{B. More general Warped products}

We now consider the more general ansatz:

$$
d s_{\lambda}^{2}=-e^{2 \alpha_{\lambda}(z)} d t^{2}+d z^{2}+e^{2 \beta_{\lambda}(z)} \sum_{i, j=1}^{d-2} \tilde{g}_{i j}(x) d x^{i} d x^{j},
$$

where $\tilde{g}_{i j}$ is independent of $t$ and $z$, and where $x=\left(x^{1}, \ldots, x^{d-2}\right)$ is an appropriate collection of additional coordinates. Again, we take the range of $z$ to include some closed interval $\left[z_{-}, z_{+}\right]$, and we confine all discussion to this interval below. This ansatz includes static spherically symmetric families of metrics $g_{\lambda}$ when $\tilde{g}_{i j}$ is the metric on the round unit sphere $S^{d-2}$.

An interesting feature of such spacetimes is that geodesics which are initially tangent to a surface $x^{i}=$ constant in fact remain in this surface. One may show that affinely parameterized null geodesics of this sort are integral curves of the vector fields $e^{-2 \alpha_{\lambda}} \partial_{t} \pm e^{-\alpha_{\lambda}} \partial_{z}$.

Thus each such null geodesic requires an affine parameter

$$
\int_{z_{-}}^{z_{+}} e^{\alpha_{\lambda}} d z
$$

to traverse the region between $z_{-}$and $z_{+}$. We see that if the two sides of the wall are to remain in causal contact (i.e., if information can flow across the wall in either direction) in the limit $\lambda \rightarrow 0$, the above quantity must be bounded uniformly in $\lambda$ for small $\lambda$. We therefore assume such a uniform bound in our treatment below.

Let us now proceed with the proof. First, we note that the consequences of condition (ii) are identical to those in section $\llbracket A$ namely, that away from $z=0$ the functions $\alpha_{\lambda}, \beta_{\lambda}$, and their first and second derivatives converge to some $\alpha_{0}, \beta_{0}$ (and their derivatives) in the limit $\lambda \rightarrow 0$. Let us consider the null convergence condition (requirement (iii)) for the null vectors

$$
\begin{aligned}
& k_{\perp}=e^{-\alpha_{\lambda}} \partial_{t}+\partial_{z}, \quad \text { and } \\
& k_{\|}=e^{-\alpha_{\lambda}} \partial_{t}+e^{-\beta_{\lambda}} \sum_{i=1}^{d-2} v^{i} \partial_{x_{i}}
\end{aligned}
$$

with $\sum_{i, j=1}^{d-2} \tilde{g}_{i j} v^{i} v^{j}=1$ and $v^{i}(x)$ independent of $t, z$ :

$$
\begin{array}{r}
0 \leq R_{a b} k_{\perp}^{a} k_{\perp}^{b}=(d-2) e^{\alpha_{\lambda}-\beta_{\lambda}} \frac{d}{d z}\left[A_{\lambda}\right], \\
0 \leq R_{a b} k_{\|}^{a} k_{\|}^{b}=e^{-\alpha_{\lambda}-(d-2) \beta_{\lambda}} \frac{d}{d z}\left[B_{\lambda}\right]+e^{-2 \beta_{\lambda}} \tilde{R} \geq 0,
\end{array}
$$

where $A_{\lambda}(z)$ and $B_{\lambda}(z)$ are again defined by (2.7) and $\tilde{R}(x)=\sum_{i, j=1}^{d-2} \tilde{R}_{i j} v^{i} v^{j}$ is defined in terms of the Ricci tensor $\tilde{R}_{i j}$ of the metric $\tilde{g}_{i j}$. Since (2.12) is identical to inequality (2.3) in section ЩA we see that, for sufficiently small $\lambda$, the functions $e^{-\beta_{\lambda}}$ and $A_{\lambda}$ are bounded both above and below on the interval $\left[z_{-}, z_{+}\right]$.

However, inequality (2.13) differs from (2.4) by the addition of the final term. Recall that our argument in section ЩA considered only the dependence on the coordinate $z$, effectively taking the $x^{i}$ to be fixed, and note that $\tilde{R}$ is a function only of the $x^{i}$. Below, we again take the $x^{i}$ to be fixed so that we may regard $\tilde{R}$ as a constant. Furthermore, it is sufficient to consider the case $\tilde{R}>0$ below, as for $\tilde{R} \leq 0$ the constraint (2.13) immediately reduces to (2.4) and the rest of the argument follows as in section IA

To show that $e^{\beta_{\lambda}(z)}$ is (uniformly) bounded under our current hypotheses, recall that we have already established that there is some positive constant $N$ such that $-A_{\lambda}<N$ for sufficiently small $\lambda$. Thus, using the explicit expression for $A_{\lambda}$, we have

$$
\frac{d}{d z}\left[e^{\beta_{\lambda}}\right]<N e^{\alpha_{\lambda}} .
$$

Integrating this equation yields

$$
e^{\beta_{\lambda}(z)}<e^{\beta_{\lambda}\left(z_{-}\right)}+N \int_{z_{-}}^{z} e^{\alpha_{\lambda}} d z^{\prime},
$$


and since the right hand side is uniformly bounded for sufficiently small $\lambda$, so is $e^{\beta_{\lambda}}$. Since $\beta_{\lambda}$ is bounded below, there in fact exists some positive constants $K_{ \pm}$such that $e^{ \pm \beta_{\lambda}}<K_{ \pm}$for sufficiently small $\lambda$.

Now, for each $x=\left\{x^{i}\right\}$, consider $z \in[-\epsilon, \epsilon]$ for any $\epsilon<\left|z_{ \pm}\right|$, where we will later take $\epsilon$ to be small. In order to provide a substitute for Corollary 1 , we wish to prove that $e^{\alpha_{\lambda}-\beta_{\lambda}}$ is bounded on $[-\epsilon, \epsilon]$ uniformly in $\lambda$ (for small $\lambda$ ). Integrating (2.13) one finds

$$
B_{\lambda}(z) \geq B_{\lambda}(-\epsilon)-\tilde{R} \int_{-\epsilon}^{z} e^{\alpha_{\lambda}+(d-4) \beta_{\lambda}} d z^{\prime}
$$

Since $B_{\lambda}(-\epsilon)$ converges in the limit $\lambda \rightarrow 0$, for small $\lambda$ we have $B_{\lambda}(-\epsilon) \geq-b_{\epsilon}$ where $b_{\epsilon}$ is some positive constant. Considering the integral in (2.16), let us note that since $e^{\alpha_{\lambda}-\beta_{\lambda}}$ is smooth, it attains some maximal value $\mathrm{M}_{\lambda}$ on the interval $[-\epsilon, \epsilon]$. Thus, the integrand $e^{(d-3) \beta_{\lambda}} e^{\alpha_{\lambda}-\beta_{\lambda}}$ is bounded (above) by $K_{+}^{d-3} \mathrm{M}_{\lambda}>0$. Multiplying the result by $e^{-(d-1) \beta_{\lambda}}<K_{-}^{d-1}$ and using $z<\epsilon$ and the definition of $B_{\lambda}$ thus yields

$$
\frac{d}{d z}\left(e^{\alpha_{\lambda}(z)-\beta_{\lambda}(z)}\right) \geq-K_{-}^{d-1} b_{\epsilon}-2 \epsilon \tilde{R} K_{-}^{d-1} K_{+}^{d-3} \mathrm{M}_{\lambda} .
$$

For the next step, it will be convenient to define $f_{\lambda}=e^{\alpha_{\lambda}-\beta_{\lambda}}$, the function with which we are currently concerned. Furthermore, we take $z_{0}$ to be the point in $[-\epsilon, \epsilon]$ where $f_{\lambda}\left(z_{0}\right)=\mathrm{M}_{\lambda}$. If $z_{0}<\epsilon$, then by the mean value theorem the derivative $\frac{d}{d z} f_{\lambda}$ attains the value $\frac{f_{\lambda}(\epsilon)-\mathrm{M}_{\lambda}}{\epsilon-z_{0}}$ somewhere between $z_{0}$ and $\epsilon$, so that this value must also satisfy the bound (2.17). But, since $z_{0}>-\epsilon$ we have

$$
\frac{f_{\lambda}(\epsilon)-\mathrm{M}_{\lambda}}{2 \epsilon} \geq \frac{f_{\lambda}(\epsilon)-f_{\lambda}\left(z_{0}\right)}{\epsilon-z_{0}} \geq-K_{-}^{d-1} b_{\epsilon}-2 \epsilon \tilde{R} K_{-}^{d-1} K_{+}^{d-3} \mathrm{M}_{\lambda} .
$$

Choosing $\epsilon^{2}<1 / 4 \tilde{R} K_{-}^{d-1} K_{+}^{d-3}$, a bit of algebra yields

$$
\mathrm{M}_{\lambda} \leq \frac{f_{\lambda}(\epsilon)+2 \epsilon K_{-}^{d-1} b_{\epsilon}}{1-\tilde{R} K_{-}^{d-1} K_{+}^{d-3} 4 \epsilon^{2}} .
$$

Note that (2.19) also holds for the remaining case where $f_{\lambda}$ attains the value $\mathrm{M}_{\lambda}$ only at $z=\epsilon$.

Finally, since $f_{\lambda}(\epsilon)$ converges as $\lambda \rightarrow 0$, the right hand side is bounded uniformly in $\lambda$ for small $\lambda$. We see that $\mathrm{M}_{\lambda}$ and thus $e^{\alpha_{\lambda}-\beta_{\lambda}}$ (on $[-\epsilon, \epsilon]$ ) has a $\lambda$-independent upper bound for small $\lambda$. In summary, we have shown:

Lemma 3: Given $\alpha_{\lambda}, \beta_{\lambda}$ satisfying (2.12), (2.13), and the stated convergence properties with respect to $\lambda$, there is an $\epsilon>0$ such that, for sufficiently small $\lambda$, the functions $e^{-\beta_{\lambda}}$ and $e^{\alpha_{\lambda}-\beta_{\lambda}}$ are bounded (uniformly in $\lambda$ ) on the interval $[-\epsilon, \epsilon]$.

It is now straightforward to prove continuity of the induced metric. As above, we consider only the interval $[-\epsilon, \epsilon]$ and the case $\tilde{R}>0$. By Lemmas 2 and 3 , for sufficiently small $\lambda$, the function $\beta_{\lambda}^{\prime}=-A_{\lambda} e^{\alpha_{\lambda}-\beta_{\lambda}}$ is bounded uniformly in $\lambda$ (for small $\lambda$ ) so that $\beta_{0}$ is continuous. In addition, we have

$$
\frac{d}{d z}\left[B_{\lambda}\right] \geq-e^{\alpha_{\lambda}+(d-4) \beta_{\lambda}} \tilde{R} \geq-e^{\alpha_{\lambda}-\beta_{\lambda}} K_{+}^{d-3} \tilde{R} \geq-K_{+}^{d-3} \tilde{R} \mathrm{M}_{\lambda}
$$

for sufficiently small $\lambda$. Thus for $z \in[-\epsilon, \epsilon]$ we have

$$
B_{\lambda}(\epsilon)+2 \epsilon K_{+}^{d-3} \tilde{R} \mathrm{M}_{\lambda} \geq B_{\lambda}(z) \geq B_{\lambda}(-\epsilon)-2 \epsilon K_{+}^{d-3} \tilde{R} \mathrm{M}_{\lambda} .
$$

The convergence of $B_{\lambda}( \pm \epsilon)$ as $\lambda \rightarrow 0$ and Lemma 3 then guarantee that on $[-\epsilon, \epsilon]$, the function $B_{\lambda}=$ $e^{(d-1) \beta_{\lambda}} \frac{d}{d z}\left(e^{\alpha_{\lambda}-\beta_{\lambda}}\right)$ satisfies a bound which is uniform in $\lambda$ for small $\lambda$. Multiplying by $e^{-(d-1) \beta_{\lambda}}$ and using Lemma 3 once again, the same must be true of $\frac{d}{d z}\left(e^{\alpha_{\lambda}-\beta_{\lambda}}\right)$. Thus $e^{\alpha_{0}-\beta_{0}}$ is continuous. Since we have already established continuity of $e^{\beta_{0}}$, we in fact have:

Theorem 2: Consider a one-parameter family of smooth metrics of the form (2.9) satisfying conditions (ii) and (iii) from section [I null geodesics along surfaces of constant $x^{i}$ in the limiting spacetime $\left(M, g_{0}\right)$ reach (and cross) the wall in finite affine parameter, then the limiting metric $g_{0}$ defines continuous induced metrics on the hypersurfaces of constant $z$. 


\section{More degrees of freedom}

Thus far, we have investigated only metric ansätze having two free functions. However, it is also straightforward to consider metrics of the form:

$$
d s_{\lambda}^{2}=-d t^{2}+d z^{2}+\sum_{i=1}^{d-2} e^{2 \beta_{\lambda}^{i}(z)}\left(d x^{i}\right)^{2}
$$

Note that we have required the existence of a timelike Killing vector field with constant norm. As usual, we take the range of $z$ to include $\left[z_{-}, z_{+}\right]$, and we take this coordinate to label the hypersurfaces $\Sigma^{z}$. Since no redshift factor is allowed in (2.22), such metrics are perhaps of less physical interest than those considered earlier. Nonetheless, we present a short proof for this case in the hopes that it will prove useful for future investigations.

The techniques developed in section $1 \mathrm{~A}$ apply in a straightforward way. It is sufficient to consider the null vectors

$$
k_{i}=\partial_{t}+e^{-\beta_{\lambda}^{i}} \partial_{x_{i}}
$$

for which the null convergence condition implies

$$
0 \leq R_{a b} k_{i}^{a} k_{i}^{b}=-\beta_{\lambda}^{i^{\prime \prime}}-\beta_{\lambda}^{i^{\prime}}\left(\sum_{k=1}^{d-2} \beta_{\lambda}^{k}\right)^{\prime} .
$$

Repeating the arguments of section $[\mathrm{A}]$ one immediately finds that $\beta_{\lambda}^{i}$ has no local minima on $\left[z_{-}, z_{+}\right]$and thus is bounded below uniformly in $\lambda$ for sufficiently small $\lambda$.

Furthermore, introducing $B_{\lambda}^{i}:=-\beta_{\lambda}^{i{ }^{\prime}} e^{\sum_{k=1}^{d-2} \beta_{\lambda}^{k}}$, one finds that (2.24) simplifies to $0 \leq \frac{d}{d z}\left[B_{\lambda}^{i}\right]$. Thus, repeating our standard argument, $B_{\lambda}^{i}$ is bounded uniformly in $\lambda$ for small $\lambda$. Together, these facts imply a uniform bound on $\beta_{\lambda}^{i \prime}=-B_{\lambda}^{i} e^{-\sum_{k=1}^{d-2} \beta_{\lambda}^{k}}$ for small $\lambda$, which in turn implies continuity of $\beta_{0}^{i}$ in the limit. We thus verify our conjecture for the special case defined by the ansatz (2.22).

\section{A RESULT WITHOUT SYMMETRY}

In this section we derive a result which ensures continuity of the induced metric across $\Sigma^{0}$ without specifying an ansatz for $g_{\lambda}$ and without imposing symmetry. However, to achieve this we introduce a number of assumptions concerning the behavior of null geodesics in the spacetime $\left(M, g_{0}\right)$. Our result below (Theorem 3 ) states roughly that it is impossible for the induced metric to be discontinuous across a positive energy wall unless some other pathology also occurs.

To this end, we begin with a definition which introduces a notion of convergence for a family $C_{\lambda}$ of geodesic congruences associated with the spacetimes $\left(M, g_{\lambda}\right)$.

Definition 1: Consider a family of spacetimes $\left(M, g_{\lambda}\right)$ satisfying condition (i) and let $C_{\lambda}$ be a geodesic congruence in $\left(M, g_{\lambda}\right)$. We then say that $C_{\lambda}$ converges to a collection of curves $C_{0}$ when the following conditions hold.

a) Note that each congruence $C_{\lambda}$ consists of a set of geodesics $\left\{\gamma_{\lambda}^{x}\right\}$ for $x$ in some appropriate label space $X$. We require that there be a choice of affine parameter $s$ along each curve $\gamma_{\lambda}^{x}$ such that, for each $x$ in the label set $X$, the affine parameter ranges over the interval $[0,1]$ and the maps $\gamma_{\lambda}^{x}(s):[0,1] \rightarrow M$ converge pointwise in the limit $\lambda \rightarrow 0$ to some map $\gamma_{0}^{x}(s):[0,1] \rightarrow M$.

b) Away from the hypersurface $\Sigma^{0}$, we require that the first and second derivatives of $\gamma_{\lambda}^{x}(s)$ with respect to both $x$ and $s$ must converge to the corresponding derivatives of $\gamma_{0}^{x}(s)$, which we also require to be well-defined. Thus, away from $\Sigma^{0}$, we see that $\gamma_{0}^{x}$ is also a geodesic of $\left(M, g_{0}\right)$ and that $s$ is again an affine parameter.

We denote the collection of functions $\gamma_{0}^{x}:[0,1] \rightarrow M$ by $C_{0}$.

As defined above, $C_{0}$ is merely a collection of curves and need not form a congruence. For example, it is possible that all of the curves in $C_{0}$ might coincide. However, consider the case where the $C_{\lambda}$ are hypersurface orthogonal null 
congruences (without caustics) and where the spacetimes $\left(M, g_{\lambda}\right)$ satisfy the null convergence condition (iii). Then by the Raychaudhuri equation, the expansion $\theta$ is non-increasing along each geodesic in the congruence (see e.g., [13]). But the expansion is determined by mixed second derivatives of the functions $\gamma_{\lambda}^{x}(s)$ with respect to $x$ and $s$ together with the metric $g_{\lambda}$ and its first derivative. As a result, whenever $\theta_{0, x}$ is well-defined the expansion must converge in the limit $\lambda \rightarrow 0$ to the associated expansion $\theta_{0, x}$ of $\gamma_{0}^{x}(s)$. Thus, in any interval where it is well-defined, the expansion of $\gamma_{0}^{x}(s)$ is again a non-increasing function of $s$. Furthermore, since the area elements carried by the congruences $C_{\lambda}$ converge to the area element carried by $C_{0}$, the expansion $\theta_{0, x}$ can cease to be well-defined only at $\Sigma^{0}$ or when $\theta_{0, x} \rightarrow-\infty$ at some affine parameter $s_{0}$.

Suppose then that $\gamma_{0}^{x}$ is not in $\Sigma^{0}$ at $s=1$ and that $C_{0}$ does form a congruence near $s=1$, so that the expansion $\theta_{0, x}$ is well-defined there. Then, since $\left.\theta_{\lambda, x}\right|_{s=1}$ converges as $\lambda \rightarrow 0$ to $\left.\theta_{0, x}\right|_{s=1}$, for sufficiently small $\lambda$, the expansions $\left.\theta_{\lambda, x}\right|_{s=1}$ are bounded uniformly in $\lambda$. But since each $\theta_{\lambda, x}(s)$ is a non-increasing function, this means that such $\theta_{\lambda, x}(s)$ are bounded below uniformly in both $\lambda$ and $s$. As a result, the limiting expansion $\theta_{0, x}(s)$ associated with $\lambda=0$ is also bounded below and does not diverge to $-\infty$.

Similarly, if $\left.\gamma_{0}^{x}\right|_{s=0}$ is not in $\Sigma^{0}$ and if $C_{0}$ forms a congruence near $s=0$, then $\theta_{0, x}(s)$ is bounded above. If both of these conditions hold, then the expansion $\theta_{0, x}(s)$ is well-defined everywhere away from $\Sigma^{0}$ and is bounded.

It is useful to summarize this discussion in the following Lemma:

Lemma 4: Suppose that $C_{\lambda}$ are hypersurface-orthogonal null congruences in the spacetimes $\left(M, g_{\lambda}\right)$ which converge to the set of curves $C_{0}$ in $\left(M, g_{0}\right)$ in the sense of Definition 1. Suppose also that $\left.\gamma_{0}^{x}\right|_{s=0},\left.\gamma_{0}^{x}\right|_{s=1} \notin$ $\Sigma^{0}$ and that $C_{0}$ forms a congruence near $s=0$ and near $s=1$. If the spacetimes $\left(M, g_{\lambda}\right)$ satisfy the null convergence condition (iii), then the expansion of $C_{0}$ is well-defined away from $\Sigma^{0}$ and is bounded along each curve in $C_{0}$.

Now, we wish to show that the induced metric must be continuous across $\Sigma^{0}$ unless some additional pathology arises in the spacetime. Stating this precisely will also require us to define a notion of the induced metric $h$ on each side of the hypersurface $\Sigma^{0}$ in the spacetime $\left(M, g_{0}\right)$. We do so as follows:

Definition 2: Consider a singular spacetime $\left(M, g_{0}\right)$ defined as a limit of smooth spacetimes $\left(M, g_{\lambda}\right)$ satisfying condition (i). Furthermore, consider any smooth foliation of the manifold $M$ near the singular hypersurface $\Sigma^{0}$ which includes $\Sigma^{0}$ as a leaf. Let us label the leaves by a parameter $z$ taking $z=0$ at $\Sigma^{0}$, and referring to the leaf at $z$ as $\Sigma^{z}$.

Now, for each $z>0$ there is some metric $h_{z}$ induced by embedding $\Sigma^{z}$ in $\left(M, g_{0}\right)$. Suppose that the limit $h_{0+}:=\lim _{z \downarrow 0} h_{z}$ (in which $z$ approaches zero from the positive side) exists as a tensor field on $\Sigma^{0}$. Then we say that $h_{0+}$ is an induced metric on the $z>0$ side of $\Sigma^{0}$. We may similarly define an induced metric $h_{0-}$ on the $z<0$ side of $\Sigma^{0}$.

Note that, in general, $h_{0+}$ need not agree with $h_{0-}$; i.e., the induced metric need not be continuous across $\Sigma^{0}$. Also note that the definition above allows $h_{0 \pm}$ to depend on the choice of foliation.

We are now ready to state our theorem:

Theorem 3: Consider any spacetime $\left(M, g_{0}\right)$ satisfying (i) and (ii) from section $\prod$ and a foliation $\left\{\Sigma^{z}\right\}$ of $M$ near $\Sigma^{0}$ which induces a well-defined, invertible, Lorentz signature metric $h_{0 \pm}$ on each side of $\Sigma^{0}$. Suppose also that for each point $p \in \Sigma^{0}$ and each $\omega$ in some open set of rank $(d-2)$ antisymmetric contravariant tensors associated with the tangent space to $\Sigma^{0}$ at $p$, there is a set of curves $C_{0}$ such that

a) $C_{0}$ satisfies the conditions of Lemma 4 .

b) Some curve in $C_{0}$ passes through $p$.

c) The tensor $\omega$ is the antisymmetric product of the set of deviation vectors associated with $C_{0}$ at $p$; i.e, if $y^{a}$ are coordinates on $\Sigma^{0}$, then

$$
\omega^{a_{1}, \ldots, a_{d-2}}=\epsilon^{i_{1}, \ldots, i_{d-2}} y_{, i_{1}}^{a_{1}} \ldots y_{, i_{d-2}}^{a_{d-2}},
$$

where the derivatives are taken with respect to coordinates $x^{i}$ on the label space $X$ used to parameterize the curves in $C_{0}$. In particular, we require the right-hand side of (3.1) to be well-defined; i.e., the analogue of (3.1) for $\Sigma^{z}$ with $z \neq 0$ is continuous in $z$.

d) For each curve $\gamma_{0}^{x}$ in $C_{0}$ the point $\gamma_{0}^{x}(s)$ lies in $\Sigma^{0}$ for exactly one value of the parameter $s$. This feature provides a sense in which the wall may be considered "thin."

Then the induced metric is in fact continuous across $\Sigma^{0}$; i.e., $h_{0+}=h_{0-}$. 
The proof is straightforward. For any such $C_{0}$, consider the infinitesimal area element carried by a curve $\gamma_{0}^{x}$ lying in $C_{0}$ and running through $p$. This infinitesimal area element is the antisymmetric product of the deviations vectors analogous to (3.1), but with derivatives taken holding fixed the parameter $s$ along the curves. However, since the curves in $C_{0}$ are null away from $\Sigma^{0}$, the area of this element at any point $q \notin \Sigma^{0}$ may instead be evaluated using the deviation vectors along a surface of constant $z$. That is, one may use (3.1) on a surface $\Sigma^{z}$ containing $q$ (and thus with $z \neq 0$ ).

In particular, we may evaluate the limits of the area as $z \rightarrow 0$ from either side. The result is simply the area assigned to the corresponding rank $(d-2)$ antisymmetric tensor $\omega$ at $p$ by the limiting metric $h_{0+}$ or $h_{0-}$.

Now, by assumption, the metrics $h_{0 \pm}$ are non-degenerate. Thus, knowledge of such areas for an open set (i.e., a $(d-1)$-parameter family) of rank $(d-2)$ contravariant tensors $\omega$ on $\Sigma^{0}$ in fact determines the full induced metric ${ }^{2}$ on $\Sigma^{0}$. But these areas are continuous functions of the affine parameter by Lemma 4 , and since we have imposed that the wall is "thin" as measured by $s$, this affine parameter is a continuous function of $z$. Thus, the induced metrics on the leaves $\Sigma^{z}$ must also be continuous in $z$. In particular, we must have $h_{0+}=h_{0-}$.

\section{DISCUSSION}

In the work above, we have explored the idea that the null convergence condition might imply continuity of the induced metric across thin walls in general relativity and thus lead directly to the Israel junction conditions [7]. A full proof of this conjecture would solidify the physical basis behind Israel's formalism, whereas a counter-example would lead to a new class of physically interesting thin-wall solutions.

It is important to stress, however, that in cases where one does not require the null convergence condition we see no general physical justification for requiring continuity of the induced metric, or indeed any other simple behavior at the thin-wall singularity. Thin-wall limits of general smooth negative energy spacetimes will thus be quite complicated to describe. For example, the Riemann tensor will in general fail to be a well-defined distribution due to the non-linearities in its definition. Thus, it is not clear that the limiting thin-wall spacetime should itself satisfy any well-defined version of the Einstein equation; certainly, it need not satisfy the Israel conditions [7]. As a result, there may well be a large new class of thin-wall spacetimes not satisfying the Israel conditions 7] but which are of physical relevance to the Randall-Sundrum scenario [4, 5], as this scenario violates the null convergence condition.

Returning now to the present work, we have proven that the null convergence condition leads to continuity of the induced metric for various special cases. In section [I we considered thin walls which can be approximated by certain families of smooth warped product spacetimes; in particular, we considered approximating metrics $g_{\lambda}$ of the form

$$
d s^{2}=-e^{2 \alpha(z)} d t^{2}+d z^{2}+e^{2 \beta(z)} \sum_{i, j=1}^{d-2} \tilde{g}_{i j}(x) d x^{i} d x^{j} .
$$

The physics of this ansatz can be decomposed into a number of independent assumptions. First, we have taken the approximating spacetimes to be independent of time. While this simplifies the calculations, one expects a similar proof to go through in the general time-dependent case. The point is that, in the limit of a smooth thin wall, it is natural to require derivatives along the wall (e.g., time derivatives) to remain small compared to derivatives across the wall (which necessarily become large). Thus, allowing non-zero tangential derivatives should be only a small correction to the equations of motion when viewed relative to the large transverse derivatives.

However, other generalizations (such as allowing off-diagonal components of $g_{\lambda}$ ) may be more subtle, and seem unlikely to be tractable using only the rather elementary methods of section III One might hope to gain more control by following Israel [7] and casting the problem in terms of the metric and intrinsic curvature of some foliation. However, our attempts to date in this direction have not proven fruitful. We therefore leave a more complete analysis for further investigations.

It is interesting to contrast the use of metric ansätze (Theorems 1 and 2 in section 【I) with the approach based on null congruences used to prove Theorem 3 in section IIII On the one hand, Theorem 3 is quite powerful as it requires neither symmetries nor any other particular ansatz form for the approximating spacetimes $\left(M, g_{\lambda}\right)$. On the other hand, the reader will note that the complete list of assumptions required to prove Theorem 3 is rather long. In

\footnotetext{
2 This follows from the fact that the square of the area $A$ associated with the antisymmetric contravariant tensor $\omega$ of rank $(d-2)$ is

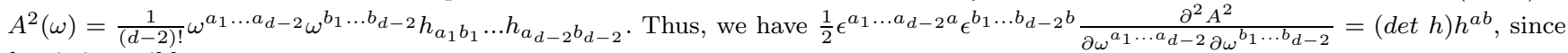
$h_{a b}$ is invertible.
} 
particular, recall that Theorem 3 assumes not only that the induced metric on each side of the wall exists as a tensor field, but also that it is invertible and of Lorentz signature. This is in contrast to the results of section 1 which prove (within their own context) that the metric on each side of the wall is well-defined.

Furthermore, the settings in section [I] include cases in which the induced metric on the thin wall is degenerate; i.e., where the wall becomes null in the limit $\lambda \rightarrow 0$. In section II we were able to show only that the function $e^{\alpha_{0}}$ is continuous at $\Sigma^{0}$, allowing $\alpha_{0}$ itself to diverge toward negative infinity as $z \rightarrow 0$, in which case the induced metric at $\Sigma^{0}$ would be degenerate. In contrast, we were able to show that the function $\beta_{0}$ is continuous at $\Sigma^{0}$, so that the corresponding metric component $e^{\beta_{0}}$ is both continuous and non-vanishing. Null walls are quite physical, and a particularly interesting example arises in thin-wall models of flat fundamental domain walls, whose stress tensor is Poincaré invariant along the wall. For example, we may note from solutions [14, 15, 16, 17] for spherical such domain walls that the walls tend to become null in the flat limit; i.e., as the radius of the sphere grows to infinite size. On the other hand, the degenerate metrics associated with null walls cannot be reconstructed from their area elements as would be required by a generalization of Theorem 3 .

Another assumption used in Theorem 3 was that one can identify an open set (in particular, a $(d-1)$-parameter family) of null geodesic congruences in each $\left(M, g_{\lambda}\right)$ such that, for each value of the $(d-1)$ parameters, the associated congruences $C_{\lambda}$ converge to a sufficiently nice family of curves $C_{0}$ in $\left(M, g_{0}\right)$. While this need not be the case for the situations considered in section II we note that all cases studied in section II do have at least one such family of congruences $C_{\lambda}$ originating on each side of the wall; namely, the congruence of null geodesics with $x^{i}=$ constant. It would be interesting to understand if this is related to the difficulty in generalizing the results of section with non-trivial redshift and more than two free functions.

\section{Acknowledgments}

The authors thank Belkis Cabrera Palmer, Andrés Gomberoff, Eric Poisson, Ricardo Troncoso, Jorge Zanelli, and especially Werner Israel for useful discussions. The original idea for this project arose in discussions between one of the authors (DM) and Belkis Cabrera Palmer. This work was supported in part by NSF grant PHY0354978 and by funds from the University of California and the Perimeter Institute for Theoretical Physics. Finally, one of the authors (SY) would like to express his gratitude to the UCSB College of Creative Studies for providing a flexible educational system, from which he benefited enormously.

[1] N. Arkani-Hamed, S. Dimopoulos and G. R. Dvali, "The hierarchy problem and new dimensions at a millimeter," Phys. Lett. B 429, 263 (1998) arXiv:hep-ph/9803315.

[2] N. Arkani-Hamed, S. Dimopoulos and G. R. Dvali, "Phenomenology, astrophysics and cosmology of theories with submillimeter dimensions and TeV scale quantum gravity," Phys. Rev. D 59, 086004 (1999) arXiv:hep-ph/9807344.

[3] I. Antoniadis, N. Arkani-Hamed, S. Dimopoulos and G. R. Dvali, "New dimensions at a millimeter to a Fermi and superstrings at a TeV," Phys. Lett. B 436, 257 (1998) arXiv:hep-ph/9804398.

[4] L. Randall and R. Sundrum, "A large mass hierarchy from a small extra dimension," Phys. Rev. Lett. 83, 3370 (1999) arXiv:hep-ph/9905221.

[5] L. Randall and R. Sundrum, "An alternative to compactification," Phys. Rev. Lett. 83, 4690 (1999) arXiv:hep-th/9906064.

[6] W. Israel, "Gravitational collapse and causality," Phys. Rev. 153, 1388 (1967).

[7] W. Israel, "Singular Hypersurfaces and Thin Shells in General Relativity," Nuovo Cimento, 44 (1966) 4349.

[8] C. Lanczos, "Bemerkung zur de Sitterschen Welt," Phys. Zeits. 23539 (1922).

[9] C. Lanczos, "Flächenhafte Verteilung der Materie in der Einsteinschen Gravitationsheorie," Ann der Phys. 74, 528 (1924).

[10] G. Darmois, "Les équations de la gravitation einsteinienne," in Mémorial des sciences mathematíques XXV(GauthierVillars, Paris, 1927).

[11] C. W. Misner and D. H. Sharp, "Relativistic equations for adiabatic spherically symmetric gravitational collapse," Phys. Rev. B136, 571 (1964).

[12] W. Israel, private communication.

[13] R. M. Wald, General Relativity (U. Chicago Press, Chicago, 1984).

[14] A. Vilenkin, "Gravitational Field Of Vacuum Domain Walls," Phys. Lett. B 133 (1983) 177.

[15] J. Ipser and P. Sikivie, "The Gravitationally Repulsive Domain Wall," Phys. Rev. D 30, 712 (1984).

[16] J. R. Ipser, "Repulsive And Attractive Planar Walls In General Relativity," Phys. Rev. D 30, 2452 (1984).

[17] A. Vilenkin and E. P. S. Shellard, Cosmic Strings and other Topological Defects (Cambridge University Press, Cambridge: 1994). 